\title{
A Centralized Model Predictive Control Strategy for Dynamic Supply Chain Management
}

\author{
Dongfei Fu*, Clara Mihaela Ionescu*, El-Houssaine Aghezzaf**, Robin De Keyser*, \\ * Department of Electrical energy, Systems and Automation, Ghent University, \\ Gent, BELGIUM (e-mails: \{Dongfei.Fu; \\ ClaraMihaela.Ionescu; Robain.DeKeyser\} @UGent.be) \\ ** Department of Industrial Management, Ghent University, \\ Gent, BELGIUM (e-mail: Elhoussaine.Aghezzaf @UGent.be)
}

\begin{abstract}
Reducing the Bullwhip effect is one of several crucial problems in supply chain management. In this paper, a centralized Model Predictive Control (MPC) strategy is applied to control inventories in a 4 echelons supply chain. The single MPC controller used in this strategy optimizes globally and finds an optimal ordering policy for each node. The controller relies on a linear discrete-time state-space model to predict process output and the prediction can be approached by two multi-step predictors, which depend on measurability of the controller states. The objective function takes a quadratic form and thus the resulting optimization problem can be solved via standard quadratic programming. Simulation results show that centralized MPC strategy can track customer demand and maintain a proper inventory position level with reduced Bullwhip effect.
\end{abstract}

Keywords: Model predictive control; Supply chains; Bullwhip effect; Multi-step predictor

\section{INTRODUCTION}

The last decades witnessed a transition of the production of industrial goods from the local or national level to facilities with global outreach that serve international markets. This development has put substantial stress on the supply chain of today's enterprises. Traditionally, supply chain management (SCM) employed heuristic techniques for the control of its real process. It is becoming increasingly difficult for the companies to compete on a global scale with only heuristic decision-making processes. As a result a systematic SCM method that maintains the inventory level at each node of supply chain to satisfy its customer demands by ordering products from the upstream node is imperative. In this paper we develop a dynamic model of a supply chain process and illustrate how centralized model predictive control is suitable for reducing Bullwhip effect.

The tendency of demand variability to increase as one moves upward in the supply chain is commonly known as bullwhip effect. There have been many methods proposed to eliminate or reduce the bullwhip (Dejonckheere et al., 2003, Disney and Towill, 2003, Lin et al., 2004). Most of these works are based on the analysis of a class of replenishment strategies known as order-up-to level policies.

Recent work utilizing model predictive control has been found to provide an attractive solution for SCM. There are several advantages of using MPC for SCM. MPC can minimize or maximize an objective function that represents a suitable measure for supply chain performance. MPC can be tuned to achieve stability and robustness in the presence of disturbance and stochastic demand. MPC was first applied to inventory management by Kapsiotis et al. (1992) for a single manufacturing site problem. It was developed subsequently and there were increasing reports on the application of MPC to SCM in the last decades. Lin et al. (2005) presented a Minimum Variance Control system with two separate setpoints for the actual inventory level and for the WIP (WorkIn-Process) level. Their MPC control strategy outperformed classical control in mitigating the Bullwhip effect. Wang et al. (2008) examined the application of MPC to inventory control problems arising in semiconductor manufacturing. Maestre et al. (2009) proposed a distributed MPC algorithm for a twonode supply chain. Alessandri et al. (2011) combined minmax optimization and MPC to solve inventory control problems of multi-echelon, multi-product distribution centre.

Previous work focused on a fully decentralized MPC strategy (Fu et al., 2012) to update ordering decision for Bullwhip reduction. One frequently suggested strategy for reducing Bullwhip effect is to centralize demand information, i.e. to make customer demand information available to every node of supply chain. The purpose of this paper is to demonstrate the applicability of a fully centralized MPC to the problem of dynamic management of supply networks. With this implementation, ordering policy for each node of supply chain member is optimized by a global coordinator. This control strategy is feasible for the problems where all nodes belong to one enterprise.

The remainder of the paper is structured as follows. In section 2 , the four nodes supply chain process is described and the discrete time controller model for the overall supply chain process is developed. Using the centralized model the two methods for predicting future process outputs are used and 
MPC algorithm is applied in section 3. Simulation results in section 4 show that an appropriate tuning of the parameters can be chosen to produce the required performance.

\section{SUPPLY CHAIN DYNAMICAL MODEL}

\subsection{Supply Chain Process Model}

Consider a supply chain consisting of all nodes involved in fulfilling a customer demand. A four nodes supply chain network is studied in this paper including a factory $F_{a}$, a distributor $D_{i}$, a wholesaler $W_{h}$ and a retailer $R_{e}$. For each node $i \in\left\{F_{a}, D_{i}, W_{h}, R_{e}\right\}$ the information and material exchange with its supplier and customer is depicted in Fig. 1.

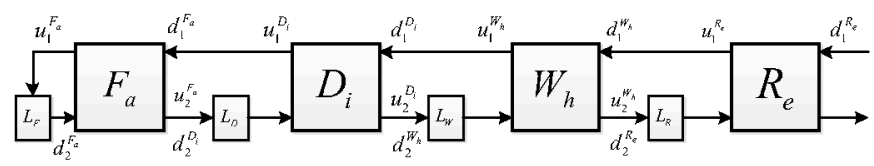

Fig. 1. A four-node supply chain

It is assumed that decisions of ordering and delivery are taken within equally spaced time periods (e.g. days, weeks or months). The duration of base time period depends on the dynamic characteristics of the network. Each node $i \in\left\{F_{a}\right.$, $\left.D_{i}, W_{h}, R_{e}\right\}$ is characterized by 3 variables $\left[I^{i}(k), I P^{i}(k), O_{*}^{i}(k)\right]$, defined as follows. The inventory level $I^{i}(k)$ is the number of products at any time instant $k$ in stock of node $i$. Inventory position $I P^{i}(k)$ is defined to better monitor the change in inventory level and it includes inventory plus products in transportation from its supplier. Standing order $O_{*}^{i}(k)$ for each node is the amount of order to be processed.

Table 1. Variable mapping for MPC controller

\begin{tabular}{l|l}
\hline Process variable & Supply chain information \\
\hline Outputs $y_{1}^{i}$ & Inventory position $I P^{i}$ \\
\hline Outputs $y_{2}^{i}$ & Standing order $O_{*}^{i}$ \\
\hline Inputs $u_{1}^{i}$ & $\begin{array}{l}\text { Amount of orders placed by node } i \\
\text { to upstream node } i+1\end{array}$ \\
\hline Inputs $u_{2}^{i}$ & $\begin{array}{l}\text { Amount of products delivered to the } \\
\text { downstream node } i-1 \text { by node } i\end{array}$ \\
\hline Disturbance $d_{1}^{i}$ & Demand of downstream node $i-1$ \\
\hline Disturbance $d_{2}^{i}$ & Delivery from upstream node $i+1$ \\
\hline Setpoint $r^{i}$ & Inventory position targets \\
\hline
\end{tabular}

This supply chain process model is not part of the control system. Its only purpose is to represent the plant in further simulations and analytical work.

\subsection{Controller Model}

The controller model provides a prediction of future supply chain process outputs as a function of manipulated variables and estimated disturbances. The controller model is part of the control system, and its states may be partially or fully known. In the present work, the MPC controller has the form of a linear discrete-time state-space model

$$
\begin{gathered}
x(k+1)=A x(k)+B \mu(k) \\
y(k)=C x(k)+D \mu(k)
\end{gathered}
$$

The input vector definition is $\mu(k)=\left[u^{T}(k) d^{T}(k) w^{T}(k)\right.$ $\left.v^{T}(k)\right]^{T}$, where the input vectors $u, d, w, v$ represent manipulated variables, load disturbances and 2 unmeasured disturbances respectively. The unmeasured disturbances are not considered in this paper but reserved for future extension of current work. The matrices $B, D$ in controller model can be partitioned by $B=\left[\begin{array}{llll}B_{u} & B_{d} & B_{w} & 0\end{array}\right], D=\left[\begin{array}{llll}0 & D_{d} & 0 & D_{v}\end{array}\right]$.

In Fig. 1, a time delay $L_{i}$ is assumed for all delivery actions together with consideration of the nominal ordering delay such that products dispatched from node $i+1$ at time $k$ will be available to node $i$ at time $k+L_{i}+1$. Take a node $i \in\left\{F_{a}, D_{i}, W_{h}, R_{e}\right\}$ of supply chain to analyse its dynamics. A material balance around any network node involves the inventory position level at time instances $k$ and $k-1$, as well as the total incoming products from upstream node and total outgoing products to downstream node and thus

$$
y_{1}^{i}(k)=\frac{1}{1-z^{-1}}\left(d_{2}^{i}(k)-u_{2}^{i}(k)\right)
$$

where $z^{-1}$ represents both of the backward shift operator and complex variable in $z$-transformation. Notice that if applied to time-dependent signal $s(k)$, it is a backward shift operator, i.e. $z^{-L} s(k)=s(k-L)$.

Dejonckheere et al. (2003) pointed to 2 types of sequence of events in supply chain operation and we choose the first type. The order information can be communicated instantaneously, but an order placed at time $k$ can only be processed at time $k+1$. Therefore, the standing order $y_{2}^{i}(k)=O_{*}^{i}(k)$ is defined:

$$
y_{2}^{i}(k)=\frac{1}{1-z^{-1}}\left(d_{1}^{i}(k)-u_{2}^{i}(k)\right)
$$

If we assume that there is always enough stock at node $i$ to meet customer demand then we have $u_{2}^{i}(k)=z^{-1} y_{2}^{i}(k)$. This is essentially a linearization assumption to simplify subsequent analysis. It follows that $d_{1}^{i}(k)=y_{2}^{i}(k)$, which means $u_{2}^{i}(k)=$ $z^{-1} d_{1}^{i}(k)$. These relations between variables indicate that delivery equals to order from node $i-1$ with one time period delay: $u_{2}^{i}(k)=z^{-1} d_{1}^{i}(k)=z^{-1} u_{1}^{i-1}(k)$. Thus model (3) can be transformed to the following (5). This discrete time model captures well the basic dynamic features of material and information flow in our supply chain process.

$$
y_{1}^{i}(k)=\frac{z^{-1}}{1-z^{-1}}\left(u_{1}^{i}(k)-d_{1}^{i}(k)\right)
$$

These models of the nodes could be reorganised to give the overall model of supply chain in state-space form (1) and (2) and it will be used as the nominal controller model. The 
whole supply chain is modelled as a four-input/four-output system. The manipulated vector corresponds to the orders in supply chain nodes, while measured disturbance represents the forecasted demand. The controlled variables/outputs are the vector of inventory position.

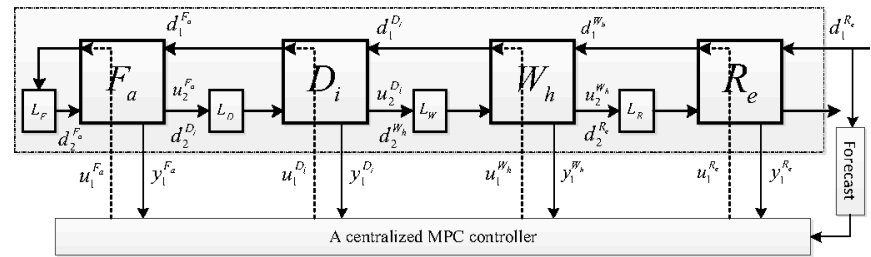

Fig. 2. Centralized control scheme for supply chain operation

We propose to apply a fully centralized MPC strategy (Fig. 2) to the supply chain described above (in squared box). If all the facilities are owned by the same company, inventory position level and demand may be shared easily. Therefore, centralized control scheme is appropriate. All available information is fed to the controller and the ordering decisions $\left\{u_{1}^{R_{e}}(k), \cdots u_{1}^{F_{a}}(k)\right\}$ are determined by a global coordinator.

\section{MPC CONTROL STRATEGY}

MPC is a control strategy based on the explicit on-line use of a process model to calculate predictions of the future process output and to optimize future control actions over a period of time. The methods for predicting future process output can be approached by two ways depending on whether the system states are measurable or not. If the state of controller model is measurable as in the case of our model, the multi-step predictor can be developed from state space model per (7). However, the predictor has to be obtained by state estimation when the states are not fully measurable. The two approaches are given in section 3.1 and 3.2 respectively.

\subsection{Multi-step Predictor Based on Measured State}

Based on the fact that past and present control actions affect the future output of the process, a receding horizon principle (De Keyser, 2003) is selected. At each time instant $k$ process model (1) (2) is used to predict future output $y(k+j \mid k)$, where $j=1, \ldots N_{2}$ and $N_{2}$ is the prediction horizon.

When the system state can be fully measured we consider MPC controller model in (1) and (2), which has the following linear discrete-time state-space form (Lee et al., 1994)

$$
\begin{gathered}
x(k)=A x(k-1)+B_{u} u(k-1)+B_{d} d(k-1)+B_{w} w(k-1) \\
y(k)=C x(k)+D_{d} d(k)+D_{v} v(k)
\end{gathered}
$$

The input vectors $u, d$ and $w$ represent ordering decision for each node, end customer demand forecast as load disturbance and un-forecasted demand. The output $y$ is a vector of measured inventory position, which may be corrupted by measurement noise. It is convenient if we lump the effect of load disturbance on the system state i.e. $D_{d}=0$.

The following optimal multi-step prediction equation can be developed by iterating difference form of model (6) and (7):

$$
\begin{aligned}
y(k)=S^{\Delta x} \Delta x(k)+I^{y} y(k \mid k) \\
+S^{\mathcal{D}} \Delta \mathcal{D}(k)+S^{\Delta u} \Delta \mathcal{U}(k)
\end{aligned}
$$

where $\mathcal{Y}(k)=\left[\begin{array}{lll}y^{T}(k+1 \mid k) & \cdots & y^{T}\left(k+N_{2} \mid k\right)\end{array}\right]^{T}$ is process output prediction over the prediction horizon $N_{2}$ based on measurement at time $k, \Delta \mathcal{U}(k)=\left[\Delta u^{T}(k \mid k) \quad \Delta u^{T}(k+1 \mid k)\right.$ ․ $\left.\Delta u^{T}\left(k+N_{u}-1 \mid k\right)\right]^{T}$ represents the future change of control moves over control horizon $N_{u}$ and we also allow the flexibility of suppressing the last $N_{2}-N_{u}$ input moves $a$ priori (i.e. $\Delta u\left(k+N_{u} \mid k\right)=\cdots \Delta u\left(k+N_{2}-1 \mid k\right)=0$ ). The measured disturbance $\Delta \mathcal{D}(k)=\left[\begin{array}{lll}\Delta d^{T}(k) & \Delta d^{T}(k+1 \mid k) & \cdots\end{array}\right.$ $\left.\Delta d^{T}\left(k+N_{u}-1 \mid k\right)\right]^{T}$ corresponds to the forecast of demand over the control horizon $N_{u}$. It explains how forecast of customer demand (measured disturbance) influences the predicted inventory position (output). In SCM context, taking use of forecasted demand in the control algorithm is a significant contributor to improved performance. They relate to output through the following dynamic matrices:

$$
\begin{aligned}
& S^{\Delta x}=\left[\begin{array}{llll}
(C A)^{T} & \left(C A^{2}+C A\right)^{T} \quad \cdots \quad\left(\sum_{j=1}^{N_{2}} C A^{j}\right)^{T}
\end{array}\right]^{T}, \\
& S^{\mathcal{D}}=\left[\begin{array}{cccc}
C B_{d} & 0 & \cdots & 0 \\
\vdots & \vdots & \vdots & \vdots \\
\sum_{j=1}^{N_{u}} C A^{j-1} B_{d} & \sum_{j=1}^{N_{u}-1} C A^{j-1} B_{d} & \cdots & C B_{d} \\
\vdots & \vdots & \vdots & \vdots \\
\sum_{j=1}^{N_{2}} C A^{j-1} B_{d} & \sum_{j=1}^{N_{2}-1} C A^{j-1} B_{d} & \cdots & \sum_{j=1}^{N_{2}-N_{u}+1} C A^{j-1} B_{d}
\end{array}\right] \\
& I^{y}=\left[\begin{array}{llll}
I & I & \cdots & I
\end{array}\right]^{T}, \\
& S^{\Delta U}=\left[\begin{array}{cccc}
C B_{u} & 0 & \cdots & 0 \\
\vdots & \vdots & \vdots & \vdots \\
\sum_{j=1}^{N_{u}} C A^{j-1} B_{u} & \sum_{j=1}^{N_{u}-1} C A^{j-1} B_{u} & \cdots & C B_{u} \\
\vdots & \vdots & \vdots & \vdots \\
\sum_{j=1}^{N_{2}} C A^{j-1} B_{u} & \sum_{j=1}^{N_{2}-1} C A^{j-1} B_{u} & \cdots & \sum_{j=1}^{N_{2}-N_{u}+1} C A^{j-1} B_{u}
\end{array}\right] .
\end{aligned}
$$

Here $\Delta$ operator represents the change in the variable from the previous sampling time (i.e. $\Delta *(k) \triangleq *(k)-*(k-1))$.

\subsection{Multi-step Predictor Based on State Estimation}

It is often highly unrealistic to assume all states of the system and disturbances are measurable. When the measurement of the whole state vector is unavailable, an estimator must be used. We assume that future unmeasured disturbances will be zero and use the nominal model (6) and (7) to estimate the future state of the process

$$
\begin{gathered}
\hat{x}(k+1 \mid k)=A \hat{x}(k \mid k-1)+B_{u} u(k)+B_{d} d(k)+K \hat{e}(k \mid k) \\
\hat{y}(k \mid k-1)=C \hat{x}(k \mid k-1)+D_{d} d(k)
\end{gathered}
$$

where $\hat{x}(k+1 \mid k)$ is the estimate of the state at future time period $k+1$ based on information available at period $k$, 
$\hat{y}(k \mid k-1)$ is the estimate of the process output at time period $k$ based on information at period $k-1, \hat{e}(k \mid k)$ is innovation term of the estimator error $\hat{e}(k \mid k)=\tilde{y}(k)-\hat{y}(k \mid k-1)(\tilde{y}(k)$ is the process output) to account for unmeasured disturbances. $K$ is a constant gain matrix and Lee et al. (1994) recommended that is set equal to Kalman filter gain.

We have obtained the measurements of the process output $\tilde{y}(k)$ and the measured disturbance $d(k)$ at the start of time period $k$, and calculated the state estimate $\hat{x}(k \mid k-1)$ and estimator error $\hat{e}(k \mid k)$ from (9) and (10). The control problem is to find optimal ordering polices $u(k)$, which will be sent to the supply chain nodes. In order to determine whether a given choice of $u(k)$ is the best, we must be able to predict its effect on plant output. This is done for a prediction horizon $N_{2}$ sampling periods. The prediction over the horizon can be approached by (9) and (10). Because the controller contains models for all nodes and the anticipation of future demand, good performance can be expected.

The optimal value of the manipulated variables at time period $k+j$ is $u(k+j \mid k)$, where $j=0, \cdots N_{u}-1$. The future values of measured disturbances $d(k)$ can be structured wisely. For simplicity, $d(k)$ and $\hat{e}(k \mid k)$ are assumed to be:

$$
\hat{e}(k+j \mid k)=\hat{e}(k \mid k) \quad j=1, \cdots N_{2}
$$

Under the same condition that we lump the effect of load disturbance on the system state i.e. $D_{d}=0$, then the multistep prediction equation can be developed by recursively using (9) and (10) while considering the assumptions (11).

$$
y(k)=S^{u} u(k)+S^{x} \hat{x}(k \mid k-1)+S^{d} d(k \mid k)+S^{e} \hat{e}(k \mid k)
$$

where $y(k)$ is the process output prediction based on the measurements until time period $k, y(k)=\left[\hat{y}^{T}(k+1 \mid k) \quad \cdots\right.$ $\left.\hat{y}^{T}\left(k+N_{2} \mid k\right)\right]^{T}$ and $u(k)$ is a vector of future manipulated variables, $u(k)=\left[\begin{array}{lll}u^{T}(k \mid k) & \cdots & u^{T}\left(k+N_{u}-1 \mid k\right)\end{array}\right]^{T}$ and the last three terms in (13) are known at the start of period $k$. The pulse response matrix $S^{u}$ and the other matrices $S^{x}, S^{e}$ are given by the following matrices. Note that $S^{d}$ is not presented here and it has the similar elements as in $S^{u}$ with substitution of $B_{d}$ for $B_{u}$.

$$
\begin{aligned}
S^{u} & =\left[\begin{array}{cccc}
C B_{u} & 0 & \cdots & 0 \\
C A B_{u} & C B_{u} & \cdots & 0 \\
\vdots & \vdots & \vdots & \vdots \\
C A^{N_{u}-1} B_{u} & \cdots & \cdots & C B_{u} \\
\vdots & \vdots & \vdots & \vdots \\
C A^{N_{2}-1} B_{u} & C A^{N_{2}-2} B_{u} & \cdots & \sum_{j=1}^{N_{2}-N_{u}+1} C A^{j-1} B_{u}
\end{array}\right], \\
S^{x} & =\left[\begin{array}{llll}
(C A)^{T} & \left(C A^{2}\right)^{T} & \cdots & \left.\left(C A^{N_{2}}\right)^{T}\right]^{T},
\end{array}\right. \\
S^{e} & =\left[\begin{array}{llll}
(C K)^{T} & (C(A+I) K)^{T} & \cdots & \left(C\left(\sum_{k=1}^{N_{2}} A^{k-1}\right) K\right)^{T}
\end{array}\right]^{T} .
\end{aligned}
$$

Taking use of forecasted demand in the output prediction for supply chain control algorithm is important for achieving good performance.

\subsection{Algorithm Calculation}

The predicted process output $y(k)$ depends on past inputs and outputs as well as the future control scenario $U(k)$. The MPC algorithm tries to calculate this control scenario by minimizing a specified objective function of any form in general. At each time period $k$, the MPC controller for SCM considers the previous information on inventory position of each node, actual customer demand, order of each node and future information on inventory position setpoint, forecasted demand to calculate a sequence of current and future order decisions on the basis of the following objective function:

$$
\begin{aligned}
& \min _{\Delta u(k+j k), j=0, \cdots, N_{k}-1} \mathcal{V}(k)=\underbrace{\sum_{j=1}^{N_{k}}\|\Delta u(k+j-1 \mid k)\|_{P(j)}^{2}}_{\text {penalty on changes of order }} \\
& +\underbrace{\sum_{j=1}^{N_{2}}\|[y(k+j \mid k)-r(k+j \mid k)]\|_{Q(j)}^{2}}
\end{aligned}
$$$$
\text { keep inventory position at setpoint }
$$

where $r(k+j \mid k)$ is the inventory position reference vector for time $k+j$ projected at time $k, Q(j), P(j)$ are penalty weights on control error and move size respectively, which enable the controller to satisfy inventory position setpoint tracking, adjust order variability. The small variation of demand at retailer end will be amplified to the upstream end, which is known as Bullwhip effect. Penalizing excess change of the manipulated variables is conducive to reducing the Bullwhip effect. In addition, suppression of excess movement of the manipulated variables leads to smoothing ordering pattern and thus results in reduced variability on factory starts The objective function can be described in vector form:

$$
\min _{\Delta \mathcal{U}(k)} \mathcal{V}(k)=\|[\mathcal{Y}(k)-\mathcal{R}(k)]\|_{\mathcal{Q}}^{2}+\|\Delta \mathcal{U}(k)\|_{\mathcal{P}}^{2}
$$

where reference $\mathcal{R}(k)=\left[\begin{array}{lll}r^{T}(k+1 \mid k), & \cdots, & r^{T}\left(k+N_{2} \mid k\right)\end{array}\right]^{T}$; $\mathcal{Q}=\operatorname{diag}\left[Q(1), \cdots, \quad Q\left(N_{2}\right)\right]$ and $\mathcal{P}=\operatorname{diag}\left[P(1), \cdots, \quad P\left(N_{u}\right)\right]$

The purpose of the control is to guide the process output from current value $\tilde{y}(k)$ to its setpoint $w(k)$ along the reference trajectory $\left\{r(k+j \mid k), j=1, \cdots N_{2}\right\}$ over the prediction horizon. Because the demand is changing every time period and the inventory position setpoints need to be adapted and updated to an economic level, where customer's demand can be satisfied while inventory holding cost should be reduced. A $1^{\text {st }}$ order reference trajectory (De Keyser, 2003) is chosen and the setpoints are updated according to

$$
w(k)=\hat{D}^{L+1}(k)+n \hat{\sigma}^{L+1}(k)
$$

where $\hat{D}^{L+1}(k)$ is an estimate of the mean lead time demand ( $\left.\hat{D}^{L+1}(k)=(L+1) \hat{D}(k)\right), \quad \hat{\sigma}^{L+1}(k)$ is an estimate of the standard deviation of forecast demand over $L+1$ period $\left(\hat{\sigma}^{L+1}(k)=\sqrt{L+1} \hat{\sigma}(k)\right)$, and $n$ is a constant chosen to meet 
a desired service level. The forecasted demand $\hat{D}(k)$ can be approached by moving average or exponential smoothing method.

\subsection{Optimization Problem Solution}

The control of supply chain is now formulated as an optimization problem in which the control moves $U(k)$ are computed on the basis of (14) subject to the linear inequality constraints. Many practical requirements in SCM may be appropriately posed as constraints on the process variables.

a). Output variable constraints. The controller minimizes the deviation of inventory position of each node from their set points. But the inventory position can only stay within high and low limit due to capacity constraints. To avoid infeasible solution in the QP, the constraints on output are implemented as soft constraints in the objective function, which follows a commonly used technique in practice for addressing this problem.

$$
\mathcal{Y}_{\min }(k) \leq \mathcal{Y}(k) \leq \mathcal{Y}_{\max }(k)
$$

b). Manipulated variable rate constraints. These are hard low and high bounds for change (move) on order of each node. If proper constraints on change of order are applied, demand variation reduction can be expected within supply chain and thus result in less fluctuation in factory thrash.

$$
|\Delta \mathcal{U}(k)| \leq \Delta \mathcal{U}_{\max }(k)
$$

where $\Delta \mathcal{U}(k)$ is defined by $\Delta \mathcal{U}(k)=R_{\Delta} \mathcal{U}(k)-\delta(k)$ with $R_{\Delta}=\left[\begin{array}{ccccc}I & 0 & 0 & \cdots & 0 \\ -I & I & 0 & \cdots & 0 \\ \vdots & \vdots & \vdots & \vdots & \vdots \\ 0 & 0 & \cdots & -I & I\end{array}\right], \delta(k)=\left[u^{T}(k-1) \quad 0 \quad \cdots \quad c\right]$

c). Manipulated variable constraints. In addition to the constraints on change of order, there are some high limits on the number of order decision due to transportation capacity limitation.

$$
0 \leq \mathcal{U}(k) \leq \mathcal{U}_{\max }(k)
$$

In presence of constraints (16)-(18), the MPC control law can be solved by standard QP (Fletcher, 1981) methods based on objective function (14) and prediction equation (8) or (12).

\section{SIMULATION EXAMPLE}

\subsection{End Customer Demand}

To illustrate the benefit of centralized MPC as an ordering decision-making tool we examine it in following simulation.

$$
u_{1}^{0}(k)=\frac{1-\theta z^{-1}}{1-\phi z^{-1}} e(k)=\frac{1-0.6 z^{-1}}{1-0.8 z^{-1}} e(k)
$$

Assume that end customer demand takes the form of an ARMA time series as in eq. (19) with an average of 8 units and the forecasted demand corresponds to ten time periods moving average of actual demand. They are shown in Fig. 3.

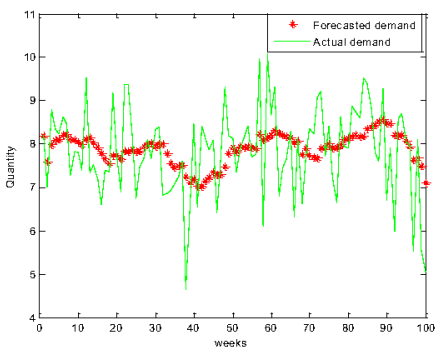

Fig. 3. Actual and forecasted customer demand

\subsection{MPC Tuning Parameters Design}

One of advantages for MPC is the flexibility to tune the parameters in the controller to meet the required performance.

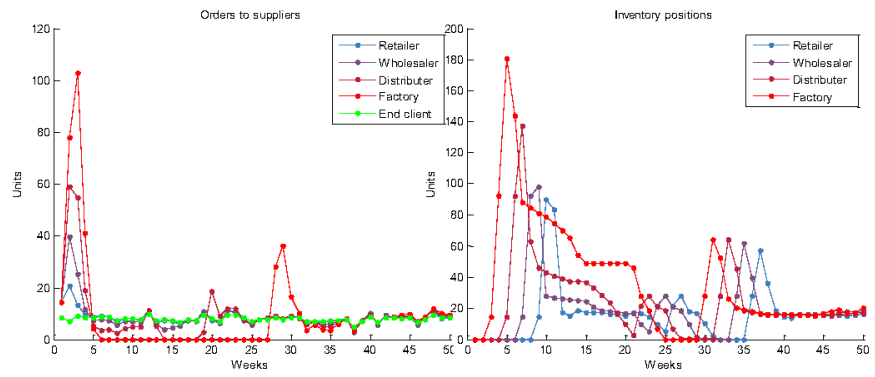

Fig. 4. Simulation results with move size weights $P(i)=0$

In this simulation example, the prediction horizon is $N_{2}=15$ while the control horizon is $N_{u}=10$, both of which exceed
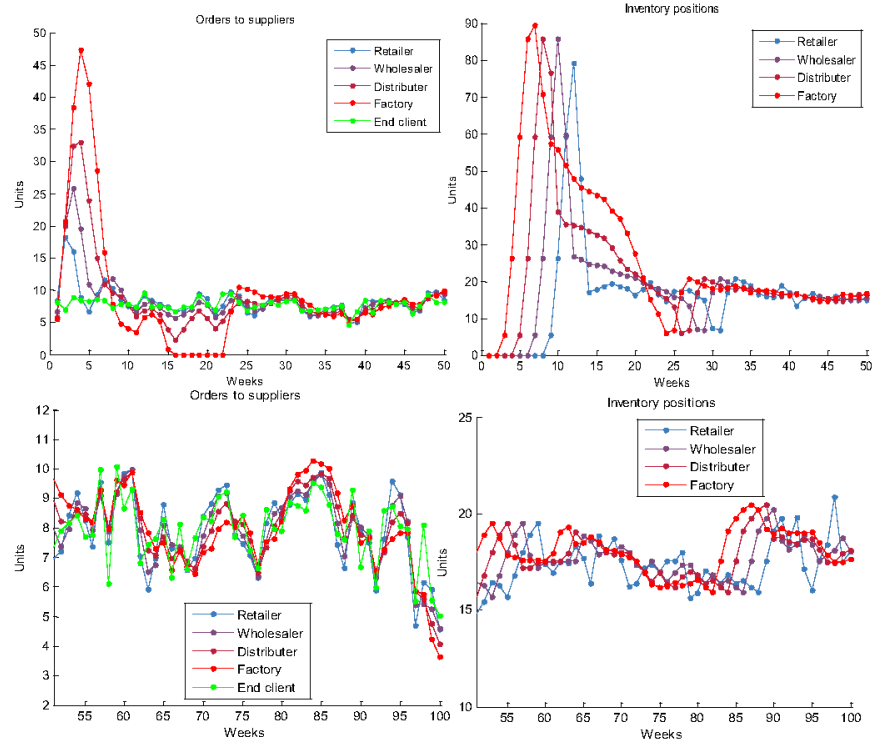

Fig. 5. Simulation results with move size weights $P(i)=I$

the collective sum of the nominal order and transportation delays for various nodes in supply chain. The long horizon is demanded by the centralized decision-making in order to perform necessary anticipative feed forward actions. The output weight $\mathcal{Q}$ is set to 1 for each controlled variable, while move suppression $\mathcal{P}$ is tuned to compare effect of different weights on Bullwhip effect reduction. No constraint 
on controlled variable and manipulated variable change is considered in the simulation.

When no penalty is applied on the move size of order in Fig.4, the ordering decisions are adjusted aggressively at first time periods and outputs keep a small fluctuation after $40^{\text {th }}$ week. The results in Fig. 4 only show the first 50 weeks. The move size weights are equal for each node in Fig. 5. The magnitude of variance on order is amplified from retailer to factory at first time periods and from lower figures we can see order decisions between week 50 and 100 keep a good tracking of end customer demand variation. The oscillation on inventory position is mainly caused by tracking the setpoints.

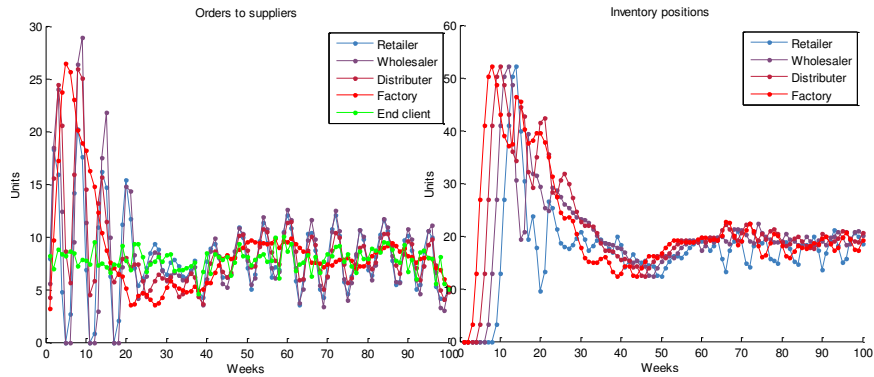

Fig. 6. Simulation results with move size

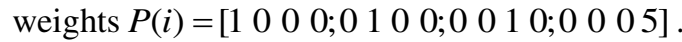

If we increase the weight on move size of factory order, then its ordering decision is smoothed and stabilized as shown in Fig. 6. This order pattern is desired because the factory thrash will not vary violently caused by demand change from very large amount to very low amount or vice versa. However, the suppression on move size of order increases the variability of inventory positions, which can be seen from week 50 to 100 .

Using the definition of Bullwhip effect proposed by Disney et al. (2003), the comparison among numerical Bullwhip quantities generated by different weights on move size and that caused by decentralized MPC strategy ordering policy and conventional ordering policies ( $\mathrm{Fu}$ et al., 2012) are shown in Table 2. They are calculated based on simulation samples rather than population.

Table 2. Bullwhip for different $P(i)$ and other strategies

\begin{tabular}{|c|c|c|c|c|}
\hline & Retailer & Wholesal. & Distribut. & Factory \\
\hline$P(i)\left(\begin{array}{llll}0 & 0 & 0 & 0\end{array}\right)$ & 1.0917 & 3.6828 & 3.3682 & 3.1186 \\
\hline$P(i)\left(\begin{array}{llll}1 & 1 & 1 & 1\end{array}\right)$ & 0.8019 & 2.1586 & 2.3092 & 2.5338 \\
\hline$P(i)\left(\begin{array}{llll}1 & 1 & 1 & 5\end{array}\right)$ & 2.5862 & 1.9115 & 0.6803 & 1.2998 \\
\hline Decentralized & 0.9888 & 2.6820 & 1.7520 & 1.5117 \\
\hline OUT & 3.4450 & 3.0731 & 2.8465 & 2.7663 \\
\hline Fractional & 2.5935 & 1.9135 & 1.3073 & 1.1106 \\
\hline
\end{tabular}

Table 2 shows that the ordering policies based on the MPC configuration outperform the conventional ordering policies in the sense of Bullwhip reduction. These results demonstrate the flexibility through centralized MPC to put different emphasis on Bullwhip suppression. When larger weight is put on factory order, it has a smooth order pattern to reduce variance of factory thrash. There is a trade-off because if a desired order rate is used then large inventory position deviations are found. From the simulation results we found the centralized MPC strategy has better customer satisfaction level than the other strategies and inventory holding profile is desired because it is made as close to zero as possible while is kept to a good level of customer satisfaction.

\section{CONCLUSIONS}

In this paper a method for determining ordering policy is derived using centralized MPC scheme. Tuning parameters play an important role in achieving desired supply chain operation performance. It has been shown that this control strategy can be tuned for different performance requirement. Good performance is observed because centralized structure has full process knowledge and signal information which allows it to coordinate the decisions in the supply chains.

\section{REFERENCES}

Alessandri, A., Gaggero, M. and F. Tonelli (2011). Min-Max and predictive control for the management of distribution in supply chains. IEEE Trans. on Control systems technology, 19(5):1075-1089.

Dejonckheere, J. Disney, S.M..Lambrecht, M.R. and Towill, D.R.(2003). Measuring and avoiding the Bullwhip Effect: A control theoretic approach. European Journal of Operational Research, volume (147), 567-590.

De Keyser R., (2003). Model Based Predictive Control. Invited Chapter in UNESCO Encyclopaedia of Life Support Systems (EoLSS). Article contribution 6.43.16.1, Eolss Publishers Co Ltd, Oxford.

Disney, S.M., Towill, D.R.(2003). On the bullwhip and inventory variance produced by an ordering policy. The International Journ. of Manag. Science, 31: 157-167.

Fletcher, R.(1981). Practical methods of optimization-volume 2. John Willey\&Sons, Ltd., U.K.

Fu, D., Dutta, A., Ionescu, C. and De Keyser, R.(2012) Reducing bullwhip effect in supply chain management by applying a model predictive control ordering policy. 14th IFAC Symposium on information control problems in manufacturing, Bucharest, Romania.

Kapsiotis, G and Tzafestas, S. (1992). Decision making for inventory / production planning using model-based predictive control. Parallel and distributed computing in engineering systems, 551-556. Elsevier, Amsterdam.

Lee, J.H., and Yu, Z.H. (1994). Tuning of model predictive controllers for robust performance. Comput. Chem. Eng., 18(1):15-37.

Lin, P.H., Jang, S.S., Wong, D.S.H. (2005). Predictive control of a decentralized supply chain unit. Industrial Engineering \& Chemistry Research, 44:9120-9128.

Lin, P.H., Wong, D.S.H., Jang, S.S., Shieh, S.S. and Chu, J.z. (2004). Controller design and reduction of bullwhip for a model supply chain using $z$-transform analysis. $J$. Process Control, volume (14), 487-499.

Maestre, J.M., Munoz de la Pena, D. and Camacho, E.F. (2009). Distributed MPC: A supply chain case study. In Joint 48th IEEE Conference on Decision and Control and 28th Chinese Control Conference, 7099-7104.

Wang, W., Rivera, D.E. (2008). A model predictive control algorithm for tactical decision-making in semiconductor manufacturing supply chain management. IEEE Trans. on Control Systems Technology, 16(5): 841-55. 